Colette FEUILLARD

Université Paris Descartes

\title{
LES NOTIONS DE CENTRE ET DE PÉRIPHÉRIE DANS LA CARACTÉRISATION DES UNITÉS LINGUISTIQUES
}

Les notions de centre et de périphérie sont liées au développement du structuralisme, en particulier par les linguistes tchèques du Cercle de Prague. La réflexion qui va suivre s'inscrit dans la continuité de ces courants. Elle se situe essentiellement sur un plan général.

L'objectif de cette étude consistera à examiner les implications théoriques et méthodologiques de ces notions et à appliquer ces dernières aux unités qui relèvent des différents niveaux de la structuration linguistique, phonèmes, morphèmes lexicaux et grammaticaux tout comme à leurs éléments constitutifs, signifiants et signifiés, ainsi qu'aux rapports syntaxiques, afin de déterminer la pertinence de cette distinction. En revanche, les relations textuelles thème rhème ne seront pas abordées. Chaque type d'unités sera analysé successivement sur le plan systémique et sur le plan discursif quand cela s'avérera possible.

\section{Implications théoriques et méthodologiques de cette distinction}

Sur un plan général et dans l'absolu, le fait de poser des éléments centraux et des éléments périphériques présuppose une hiérarchie entre les unités, des positions différentes dans le système et une incidence variable sur le plan de l'information.

D'un point de vue ontologique, le système serait donc, par essence, asymétrique et les unités de valeur inégale.

Sur le plan méthodologique, la détermination des unités centrales et périphériques exige des normes de référence systémiques et discursives, ainsi que des critères d'analyse explicites qui permettent de les identifier. Néanmoins, si l'on estime que les unités sont, par nature, centrales ou périphériques, elles devraient l'être quel que soit le système considéré (système «standard », idiolecte, parler régional, etc.) et le contexte examiné. Il y aurait donc adéquation parfaite entre les deux perspectives d'analyse, systémique et discursive. Mais qu'en est-il dans les faits ? Ce point sera abordé à partir d'exemples empruntés au français.

\section{Application de la distinction}

\subsection{Phonèmes}

\subsubsection{Analyse systémique}

Position des phonèmes dans le système :

Sont généralement analysés comme centraux les phonèmes intégrés qui possèdent des propriétés partagées par d'autres phonèmes et qui entrent dans une corrélation : c'est le cas de /p/ par opposition à /b/, par exemple. En revanche, /1/ est considéré comme périphérique, car il est le seul phonème à présenter le trait latéral. 
Mais l'étude de /l/ qui vient d'être proposée n'est pas la seule possible. Si l'on opte pour un cadre d'analyse de type générativiste, /l/ sera traité comme une consonne alvéo-dentale, distribuée, ces deux caractères se retrouvant dans d'autres unités, /t/ étant aussi alvéo-dental et /s/ présentant le trait distribué. Le phonème /1/ est alors intégré et non périphérique puisqu'il partage ces traits avec d'autres unités.

Ces deux analyses soulignent clairement le fait que tout système est une construction du linguiste. Il peut donc varier en fonction des critères sélectionnés, soit en référence à des théories différentes, soit au sein d'une même théorie.

La valeur centrale ou périphérique des phonèmes ne leur est pas inhérente. Elle résulte du point de vue choisi par le descripteur dans l'identification des propriétés des unités.

\section{Axe paradigmatique :}

D'une manière générale et sans que soit examiné dans le détail l'ensemble des oppositions et des distributions, il apparaît que le comportement phonologique de /1/ est comparable à celui des autres consonnes du français. En effet, il présente des capacités de commutation analogues à celles des phonèmes dits intégrés, bien que la fréquence des oppositions puisse varier selon les éléments considérés, leur entourage ou la position occupée dans le signifiant: /l/ s'oppose à toutes les consonnes, qu'il soit en position initiale ou en position finale devant voyelle, $\mathrm{cf}$. /lim/ « lime », /mim/ « mime »/Rim/ « rime », /sim/ « cime »/ /pal/ « pale », /pas/ « passe », /paR/ «part», /pat/ «patte», /pak/ «pack», etc. Il est également commutable, lorsqu'il est placé avant ou après une consonne, /alp/ «alpe », /arp/ « harpe », /asp/ « aspe » /pli/ «pli », /pRi/ « prix », /psi/ «psy » (abréviation de psychologue »), /pti/ «petit» (un petit, sous-entendu un petit enfant). Il entre, ainsi, dans le système des oppositions consonantiques.

\section{Axe syntagmatique :}

/l/ admet quasiment les mêmes distributions que les phonèmes dits centraux, puisqu'il peut se manifester dans toutes les positions, initiale, /lam/ «lame», médiane, /alibi/ «alibi », finale, /pil/ «pile »/, mais, à l'initiale, il ne peut être suivi d'une consonne.

\subsubsection{Analyse discursive}

Le phonème /1/, en revanche, est extrêmement fréquent dans le discours, ne serait-ce que parce qu'il est associé à l'expression de l'article défini.

A travers cet exemple, on constate donc que, outre le fait que tout système est construit et n'a pas de réalité intrinsèque mais une valeur relative, il n'y a pas de convergence systématique entre la position occupée par un phonème dans le système, selon que l'unité est considérée comme intégrée (centrale) ou non (périphérique), et son comportement fonctionnel, qu'il s'agisse des relations d'opposition avec d'autres phonèmes, de sa distribution dans les signifiants ou de sa fréquence dans le discours.

Au niveau phonologique, la non intégration d'un élément dans le système, d'où découlerait le caractère périphérique de l'unité, ne signifie pas 
nécessairement marginalité du point de vue fonctionnel ni efficacité communicative moindre, puisque /l/ est le support formel de l'article défini.

Dans le système standard, les voyelles nasales se répartissaient récemment en

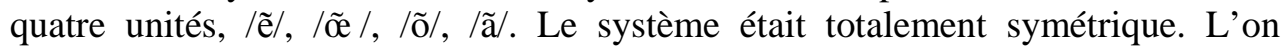
pouvait établir, en effet, une corrélation entre les voyelles orales $/ \varepsilon /$, /œ/, /o/ et /a/

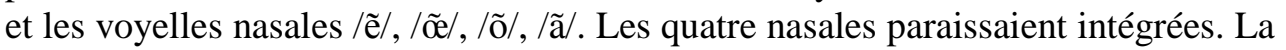
variété de français parlée dans les années 1980 en Suisse romande, dans le canton de Vaux, semblait en être une illustration (SCHOCH \& DE SPENGLER, 1980 : 106107). L'étude établissait la primauté hiérarchique de la distinction /ẽ/ /õe/ au sein des voyelles nasales. Elle avait pour objectif de vérifier l'hypothèse théorique posée par Mortéza Mahmoudian selon laquelle il y aurait corrélation entre la hiérarchisation linguistique et les dimensions mentale et sociale du langage (MAHMOUdian, 1979 : 78). Toutefois, ce qui peut valoir pour certains parlers à un moment donné ne doit en aucun cas être considéré comme définitif, ni être généralisé, notamment au français «standard» de l'hexagone. À l'heure actuelle,

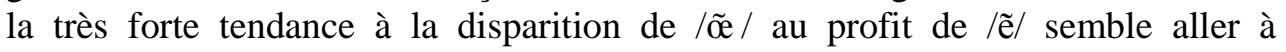
l'encontre de cette hiérarchie. Elle introduit un déséquilibre dans le système qui entraîne une dissymétrie aux dépens d'une symétrie antérieure. La voyelle /õ/ qui pouvait être considérée comme centrale en référence à la structure du système se révèle alors périphérique, puisqu'elle est de moins en moins utilisée par les locuteurs. Cette tendance à la suppression s'explique par le fonctionnement de l'unité, /õe/ n'entrant que dans un nombre très limité d'oppositions, et n'occupant pas toutes les positions.

Dans ce cas, la structure du système se trouve en conflit avec l'usage que font les locuteurs de la nasale /õ/ et c'est ce dernier qui confère, en apparence, un statut périphérique à l'unité. Il importe, cependant, de nuancer cette analyse, car tout dépend du système envisagé. En effet, dans de nombreux idiolectes, en particulier chez des locuteurs méridionaux, /õ/ est préservé.

En outre, sur le plan discursif, cette nasale supporte l'article indéfini au singulier, très fréquent dans le discours, cf. /õ bogaRsõ/ un beau garçon. Il est

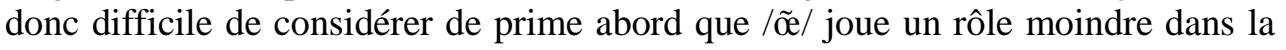
communication.

En conséquence :

2.1.3. Du point de vue des facteurs internes :

La hiérarchie peut différer : ce qui paraît central du point de vue de la structure du système peut s'avérer périphérique du point de vue du fonctionnement et inversement. Quant au fonctionnement lui-même, il n'est pas uniforme, étant donné qu'il peut y avoir divergence de comportement de l'unité en fonction des critères d'analyse qui lui sont appliqués, un faible rendement fonctionnel n'impliquant pas une fréquence faible dans le discours, cf. /ø̃e/, de même qu'il n'est pas certain qu'un rendement fonctionnel faible présuppose obligatoirement un nombre de positions limité.

2.1.4. Du point de vue des facteurs externes :

Si l'on prend en compte l'intuition du locuteur ou des critères d'ordre socioculturel (région, profession, etc.) ou générationnels (âge), de nombreuses 
variables vont intervenir en fonction de l'expérience de chacun, qui conditionnent dans une large mesure l'intuition, de sorte que le recours à cette dernière dans l'analyse des faits linguistiques peut difficilement avoir une valeur purement objective. L'intuition ne peut avoir une pertinence que si les résultats obtenus en référence à ce critère convergent avec ceux qui sont fournis par des observations strictement linguistiques.

\subsection{Morphèmes}

\subsubsection{Analyse systémique}

L'établissement d'un système «standard » en phonologie est donc loin d'être évident; il est quasiment irréalisable pour ce qui est des morphèmes, d'où la nécessité de différencier dans un premier temps les morphèmes lexicaux des morphèmes grammaticaux, étant entendu que ces deux types de classes ne sont pas clairement délimités. Les frontières sont le plus souvent perméables, car la langue se réalise sur un continuum que le linguiste est amené à scinder pour rendre compte du fonctionnement des unités dans le discours, comme le montre la distinction adverbes/prépositions, par exemple, (cf. après, il est arrivé après (adverbe), il est arrivé après son départ (préposition).

Néanmoins, d'un point de vue méthodologique, il paraît indispensable de faire une distinction entre ces deux grands types d'unités.

\section{Morphèmes lexicaux :}

Etant donné le caractère riche et ouvert du système lexical il s'avère difficile de trouver des critères susceptibles de mettre en évidence l'aspect central ou périphérique des unités. Il est impossible, en effet, de déterminer de manière absolue le degré d'intégration des morphèmes lexicaux dans le système, car tout dépend du système posé et des situations de communication envisagées. Si l'on prend les termes syntagme ou hypoténuse et qu'on les soumette à des non spécialistes de linguistique ou de mathématiques, il est évident qu'ils seront considérés comme périphériques, voire inexistants dans leur système. En revanche, il y a de fortes chances pour que syntagme devienne central pour le linguiste et hypoténuse pour le spécialiste de géométrie. Mais, en dehors de ce cas extrême qui renvoie aux langues dites de spécialité, cette difficulté se retrouve dans la langue «courante » partagée par une majorité de locuteurs. Même dans ce champ linguistique censé être commun, les zones centrales et périphériques sont fluctuantes, chacun ayant ses propres habitudes et ses termes de prédilection.

Par ailleurs, un élément jugé périphérique à un certain moment, parce que considéré comme relevant d'un usage particulier, bourgeois, littéraire, argotique, ou autre, peut devenir central à une autre époque : le terme madame était autrefois un titre honorifique réservé aux femmes des hautes classes de la société, mariées ou non (REY-DEBOVE \& REY, 2002 : 1533), alors qu'aujourd'hui il est utilisé pour désigner toute femme mariée ou censée être en âge de l'être, qu'elle le soit ou non, bien que ce dernier usage ne soit pas totalement généralisé.

Le degré d'intégration des unités est évalué empiriquement et souvent de façon intuitive, en référence aux situations de communication et à leur fréquence d'emploi dans le discours. Cependant, la fréquence n'est pas un critère fiable, car 
elle varie considérablement d'une situation à une autre. C'est la raison pour laquelle, les auteurs du français fondamental, (GOUGENHEIM, MICHEA, RIVENC, SAUVAGEOT, 1964) avaient dû recourir à un deuxième critère pour sélectionner les éléments lexicaux, la disponibilité, liée à un champ sémantique donné (cf. vêtements, repas, etc.).

Que l'on puisse poser des classes lexicales, comme celles du nom, du verbe, etc. ne change rien au problème, dans la mesure où toutes les unités constitutives d'une classe ne se définissent pas obligatoirement par rapport aux autres unités de cette classe, cf. âme et ouverture. Il est alors impossible de déterminer une hiérarchie entre ces éléments.

\section{Morphèmes grammaticaux :}

Les unités grammaticales étant en nombre plus réduit, on peut alors considérer qu'elles s'organisent en systèmes, qui correspondent aux classes grammaticales proposées par les linguistes. Ces dernières regroupent des unités qui non seulement ont un même comportement, mais qui se définissent en opposition. Les systèmes grammaticaux, comme les déterminants du nom, ont un fonctionnement relativement proche de celui des systèmes phonologiques.

Cependant, les classes grammaticales peuvent, elles aussi, varier d'un descripteur à l'autre. Ainsi, tous les linguistes ne sont pas d'accord pour distinguer temps, modes et aspects. Pour Guillaume, les formes verbales «sont toutes d'ordre temporel »(GuILlAUME, 1993 (1 $1^{\text {ère }}$ éd. 1929) : 11). Certains font du conditionnel un temps alors que d'autres l'analysent comme un mode. Cette différence de classement qui, comme pour l'analyse des phonèmes, ne résulte pas des unités elles-mêmes, mais du regard que le linguiste porte sur elles, a une incidence obligatoire sur le statut central ou périphérique qu'on peut leur attribuer. L'imparfait, si l'on admet la distinction entre temps et mode, peut avoir une valeur temporelle, considérée comme centrale, il dormait quand je suis rentrée, ou une valeur périphérique de nature modale, je voulais vous demander si je pouvais partir. L'aspect central dans le premier cas et périphérique dans le deuxième découle du fait que la valeur de passé non seulement semble la plus fréquente, ce qui demande à être vérifié, mais aussi de ce qu'elle peut se combiner avec tous les verbes, contrairement à la valeur modale qui n'est en mesure d'être actualisée que dans des contextes particuliers, notamment lorsque l'imparfait détermine un verbe modalisateur comme vouloir. Ce caractère tantôt central, tantôt périphérique de l'imparfait disparaît nécessairement si l'on ne distingue plus le temps du mode.

Si l'on observe le fonctionnement des unités indépendamment du système, c'est-à-dire de la classe à laquelle elles ressortissent, on s'aperçoit que certaines ont une distribution que l'on pourrait qualifier de complète, comme l'imparfait, le passé composé, le futur qui peuvent déterminer un verbe noyau, il viendra, ou le verbe d'une proposition subordonnée, je pense qu'il viendra, à l'inverse d'autres, qui ont une distribution soit inégale soit lacunaire. Par inégale j'entends que leur présence se manifeste plus fréquemment dans certains contextes que dans d'autres : le subjonctif est plus souvent utilisé dans une proposition subordonnée, je veux qu'il parte, qu'avec un verbe noyau de phrase, qu'il revienne vite! Les participes présent et passé tout comme le gérondif ont une distribution lacunaire 
puisqu'ils ne peuvent être employés que dans des segments subordonnés, touchant à tout ce qui se trouvait à sa portée, il a fini par faire tomber le vase.

On pourrait concevoir que les unités qui ne se combinent jamais avec le noyau d'une phrase sont plus périphériques que celles qui sont susceptibles de l'affecter. Cette analyse ne me paraît guère présenter d'intérêt, ni du point de vue opératoire ni du point de vue descriptif, la distinction entre central et périphérique étant ici redondante par rapport à la spécialisation des unités. En effet, quelle que soit la langue considérée, les unités ont tendance à se spécialiser dans certaines fonctions, à la fois par économie et pour faciliter la communication, qu'il s'agisse de la production ou de la compréhension. Il en résulte que les unités qui sont utilisées essentiellement ou exclusivement dans des propositions subordonnées sont moins fréquentes que celles qui peuvent affecter des noyaux de phrases, mais il en va de même des unités telles que l'impératif qui détermine uniquement des verbes noyaux et n'est jamais admis dans une proposition subordonnée. Dans ce cas, la fréquence ne peut pas véritablement jouer, car elle est soumise au statut de l'unité, caractéristique qui n'intervient pas dans la distinction du comportement des phonèmes. Par ailleurs, sur le plan communicatif, il est difficile d'établir une hiérarchie entre la valeur de ces unités.

Dans le cas de la polysémie, toutefois, il semble légitime de poser des valeurs centrales et des valeurs périphériques: ainsi, dans elle a une belle banane, l'allocutaire interprète prioritairement le terme banane comme désignant le fruit avant d'envisager la possibilité qu'il renvoie à un sac-ceinture ou à une coiffure. Ici, la valeur centrale est inhérente à l'unité, alors que les valeurs périphériques sont de type métaphorique. Néanmoins, il n'est pas toujours évident de dégager la valeur spécifique de l'unité. La hiérarchie repose alors sur la fréquence d'emploi. Entre les deux valeurs périphériques mentionnées précédemment, une hiérarchie s'avère également possible, l'interprétation sac-ceinture paraissant prioritaire par rapport à celle de coiffure. Mais cette hiérarchie s'explique, ici, par des facteurs relevant de l'expérience, la banane, coiffure en vogue dans les années 1960, ayant quasiment disparu, alors que le sac ceinture est encore porté.

\section{Synthèmes :}

En revanche, certaines unités synthématiques, dérivées ou composées, pourraient être interprétées comme plus ou moins centrales du point de vue de leur formation, en fonction de leur structure et de leur transparence ou de leur opacité sur le plan de la compréhension. Si l'on compare les termes eurodéputé à eurodollar, le premier manifeste un signifié transparent qui se déduit de la juxtaposition des deux membres du synthème, un eurodéputé étant un député au Parlement européen, tandis que les eurodollars sont des avoirs en dollars déposés dans une banque européenne.

2.2.2. L'examen des unités significatives, qu'elles soient lexicales ou grammaticales, tend donc à montrer que leur caractère central ou périphérique n'est plus véritablement tributaire de leur intégration plus ou moins forte au système, ce dernier devant être conçu de manière beaucoup plus lâche que dans le cas des phonèmes, avec des différences nettes entre les morphèmes lexicaux et grammaticaux. 
Par ailleurs, la distribution des éléments grammaticaux ne saurait être un facteur décisif, car elle est liée au statut fonctionnel du morphème, contrairement aux phonèmes. Enfin, la hiérarchie que l'on peut établir entre différentes valeurs au sein de la polysémie répond soit à la fréquence d'emploi de l'unité dans des situations diversifiées, soit à des facteurs d'ordre expérientiel.

\subsection{Signifiant}

Qu'elles soient lexicales ou grammaticales, les unités significatives peuvent présenter trois grands types de signifiants,

- Un signifiant invariable, cf. /tabl/ « table », /ã/ « en »,

- Un signifiant variable, mais dont les variations présentent des régularités, /fini/ « finit » /finis/ «finissent», /bwa/ «boit», /byv/ «buv(ons) », /bwav/ «boivent », /by/ «bu »,

- Un signifiant variable, à partir duquel il est impossible d'établir des règles de variation, /ctR/ «être », /syi/ « suis », /e/ « es » ou « est», /som/ «sommes », etc. Il en est de même pour avoir, aller, faire.

On pourrait émettre l'hypothèse selon laquelle les morphèmes à signifiant invariable sont mieux intégrés et donc plus centraux que les morphèmes dont la forme est irrégulière. Sur le plan de la production comme sur celui de la compréhension, le coût cognitif est, en effet, moindre. Mais si l'on recourt au critère de la fréquence, c'est l'inverse qui prévaut, tout au moins pour les morphèmes verbaux, puisque les quatre verbes les plus irréguliers sont parmi les plus fréquents.

Il est donc impossible d'établir une distinction entre éléments centraux et éléments périphériques à partir de la seule forme des unités.

\subsection{Sèmes}

Si les traits distinctifs constitutifs des phonèmes, tels que bilabial, sourd pour $/ \mathrm{p} /$, ne sont pas de type hiérarchique, en revanche les sèmes inclus dans un signifié peuvent être soit génériques soit spécifiques, ce qui peut laisser supposer une hiérarchie entre ces traits de sens : fauteuil a pour sème générique [+ siège], qui se retrouve dans tabouret, et pour sèmes spécifiques [+ individuel], [+ bras], [+ dossier]. Une première remarque s'impose, à savoir que ni [+ individuel], ni [+ bras], ni [+ dossier] ne sont spécifiques de fauteuil, [+ individuel] s'appliquant aussi à tabouret, [+ dossier] à chaise. Ce qui est spécifique, c'est la coexistence de ces trois sèmes au sein d'un même signifié en association avec le sème dit générique, [+ individuel]. En réalité, dans l'actualisation du signifié de fauteuil, ces quatre sèmes sont interdépendants et fonctionnent conjointement sans qu'il y ait un rapport hiérarchique entre eux.

Le terme stupeur qui possédait au $\mathrm{XIV}^{\text {ème }}$ siècle les sèmes [+ engourdissement $],[(+$ physique $)],[(+ \text { psychique })]^{1}$, ces deux derniers traits pouvant être co-présents ou s'exclure, a privilégié progressivement le trait [+ psychique] aux dépens du trait [+ physique] au XVIII ${ }^{\text {ème }}$. Ici, ce sont les contextes d'emploi de stupeur qui ont contribué à faire émerger le trait [+ psychique] au détriment de [+ physique] et à lui a conférer une valeur centrale en reléguant le

${ }^{1}$ Les parenthèses signifient que le trait peut ou non être sélectionné. 
trait [+ physique] dans la zone périphérique, trait qui a d'ailleurs disparu de l'usage courant. Cette hiérarchie s'est progressivement établie au cours du temps.

\subsection{Relations syntaxiques}

Le fait de concevoir les relations syntaxiques comme étant de nature hiérarchique résultant de la linéarité du discours implique nécessairement des rôles centraux et périphériques, telle l'opposition prédicat central, prédicat secondaire, subordonné. Mais ces rôles centraux, même s'ils peuvent être assumés de façon privilégiée par certaines classes (cf. le rôle prédicatif et le verbe), ne sont pas exclusivement dévolus à ces seules classes, le prédicat pouvant être, outre un verbe, travaille dans il travaille, un adjectif, insupportable, il est insupportable, un nom, il est député, etc.

La hiérarchie des rapports syntaxiques n'a pas pour corollaire obligatoire une hiérarchie des informations. Rien n'exige, par exemple, que le noyau central de la structure, le prédicat, soit l'élément le plus informatif. Ainsi, la phrase c'est sa version, avec un accent d'insistance sur $\boldsymbol{s} \boldsymbol{a}$, a pour élément central du point de vue de l'information sa qui, sur le plan syntaxique, pourrait être considéré comme périphérique, étant donné qu'il s'agit d'un déterminant grammatical du nom, qui n'est pas expansible. La hiérarchie informative des unités est assurée par la distinction thème/rhème, qui met en jeu, entre autres, l'intonation et certaines structures syntaxiques, comme les constructions clivées, c'est sa version que j'ai refusé (et non la tienne).

L'opposition centre périphérie en syntaxe pourrait enfin renvoyer à la capacité fonctionnelle d'une unité, ou plus largement à une classe d'unités, c'est-àdire à son aptitude ou non à exercer un maximum de fonctions et à recevoir des expansions multiples et variées. Ainsi, la classe du nom serait plus centrale que celle de l'adjectif, d'autant que ce dernier nécessite le nom pour fonctionner, alors que l'inverse ne joue pas.

\section{Synthèse}

Ces diverses observations font donc apparaître les caractéristiques suivantes :

3.1. Les unités linguistiques, phonèmes, morphèmes ou rôles syntaxiques, ne sont, par nature, ni centrales ni périphériques du point de vue de leur valeur informative.

3.2. Leur intégration plus ou moins forte dans le système est relative. Elle dépend du point de vue choisi par le descripteur ainsi que des critères retenus. Elle ne peut donc pas avoir de valeur véritablement opératoire, puisque l'intégration est le résultat d'une construction intellectuelle, même si celle-ci repose sur des données observables, et non le reflet direct d'une réalité linguistique. L'on sait fort bien que l'un des critères qui joue dans l'établissement du système phonologique est le principe d'économie et l'harmonie du système, ces considérations n'étant pas en soi d'ordre linguistique. 
3.3. Le rendement fonctionnel des phonèmes qui rend compte de leur capacité oppositive peut être plus ou moins élevé. Néanmoins, un moindre rendement fonctionnel ne peut en aucun cas exclure une distribution maximale et une grande fréquence d'emploi; inversement, une distribution réduite n'implique pas forcément un faible rendement fonctionnel, mais il y a de fortes chances pour que la fréquence soit limitée, surtout si l'élément considéré se trouve en position finale. Ainsi, ce qui sera susceptible de parâtre marginal du point de vue oppositionnel pourra, en revanche, ne plus l'être sur le plan discursif.

3.4. Les morphèmes en tant qu'entités significatives et indépendamment de leur fonctionnement syntaxique ne sont en mesure d'être hiérarchisés qu'en référence à la situation de communication, c'est-à-dire dans des contextes donnés (cf. langue de spécialité/langue «courante»), ou à des facteurs relevant de l'expérience. La distinction entre central et périphérique est ici largement aléatoire.

3.5. De manière analogue, sur le plan du signifiant, la régularité formelle peut être en contradiction avec la fréquence d'emploi de l'unité. Il paraît donc difficile de différencier ce qui est central de ce qui est périphérique.

3.6. Il en va de même sur le plan du signifié, bien que l'ensemble des contextes d'une unité puisse intervenir dans l'actualisation privilégiée de tel ou tel trait de sens (cf. stupeur). Ceci vaut également pour le signifié des synthèmes qui peut être plus ou moins transparent (cf. eurodéputé eurodollar).

3.7. Enfin, du point de vue syntaxique, à l'exception du rôle de prédicat conçu comme noyau de phrase, tous les autres éléments fonctionnels se trouvent de facto dans la périphérie, à des degrés divers. Par ailleurs, l'on peut se poser la question de savoir si des unités plurifonctionnelles comme les noms sont mieux intégrées que des éléments unifonctionnels tels que les articles, aussi bien dans le système que dans le discours, du simple fait que les premières ont une capacité fonctionnelle plus large que les secondes. Ces deux types d'unités en français sont solidaires et ne peuvent fonctionner l'un sans l'autre, le nom exigeant presque toujours l'article.

3.8. On pourrait supposer l'existence d'une corrélation entre la dynamique de la langue et la position périphérique des unités, en posant l'hypothèse que les unités le plus souvent en position périphérique sont vouées à plus ou moins longue échéance à disparaître du système. C'est ce que semble confirmer la tendance à

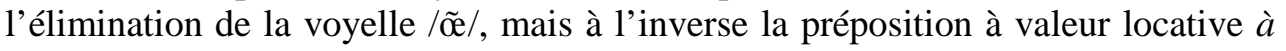
que l'on peut considérer comme occupant une position centrale dans l'expression de l'espace, compte tenu de sa valeur ponctuelle qui sert de référence aux autres positions, se trouve en concurrence avec sur et tend à être supplanté par cette dernière dans un grand nombre d'emplois, il habite à paris/il habite sur Paris.

3.9. En réalité, les faits linguistiques se heurtent constamment à des forces antagonistes qui rendent la généralisation difficile. Seules les récurrences 
permettent de dégager des tendances qui peuvent être remises en cause au fil du temps.

\section{Conclusion}

Les différentes remarques qui précèdent montrent qu'il est difficile de poser une valeur conceptuelle homogène des notions de centre et de périphérie. Les critères auxquels il est fait référence pour justifier cette distinction sont multiples et variables selon le point de vue envisagé, systémique ou discursif. De plus, les résultats obtenus en fonction des critères utilisés ne sont pas nécessairement convergents.

Il en résulte que ces notions ne sauraient avoir de valeur définitoire. Néanmoins, elles peuvent avoir une valeur descriptive dans une situation donnée, à condition que le domaine d'application soit clairement spécifié (fonctionnel, sémantique, informatif, etc.) et que les critères d'identification des éléments centraux et périphériques soient explicitement posés.

En outre, la distinction centre périphérie ne peut être dichotomique. Se situant sur un continuum, elle est nécessairement graduelle, comme l'illustre le tableau ci-dessous ${ }^{2}$ établi pour les phonèmes, qui, par ordre décroissant, iraient du centre à la périphérie :

\begin{tabular}{|c|c|c|c|}
\hline Intégration & $\begin{array}{c}\text { Rendement } \\
\text { fonctionnel }\end{array}$ & Distribution & Fréquence \\
\hline+ & + & + & + \\
\hline+ & - & + & + \\
\hline+ & - & - & + \\
\hline+ & - & - & - \\
\hline- & - & - & - \\
\hline
\end{tabular}

Les notions de centre et périphérie peuvent être, effectivement, utiles pour décrire les données linguistiques dans leur fonctionnement. Mais elles doivent être conçues dans une perspective dynamique et mouvante, ces caractéristiques n'étant attachées aux unités que dans des contextes d'emploi déterminés et non de façon permanente, encore moins définitive. De manière analogue, il ne saurait y avoir de corrélation systématique entre caractère central et stabilité des unités, ni entre caractère périphérique et variabilité, bien qu'il puisse y avoir congruence entre ces aspects.

La distinction initiale entre centre et périphérie s'inscrit historiquement dans un courant de pensée idéaliste où la langue était conçue comme un système rationnel et hiérarchisé. L'usage, en revanche, témoigne d'une grande souplesse et d'une dynamique des unités dans leur fonctionnement dont doivent rendre compte non seulement la description mais aussi les concepts opératoires posés par la théorie.

\footnotetext{
${ }^{2}$ Le tableau ne présente que certains cas de figure.
} 


\section{BIBLIOGRAPHIE}

Gougenheim Georges, Michea René, Rivenc Paul, Sauvageot Aurélien (1964), Élaboration du français fondamental, Paris, Didier.

Guillaume Gustave (1993, $1^{\text {erre }}$ éd. 1929), Temps et verbe, Paris, Honoré Champion.

MAHMOUDIAN Mortéza (1977), Dimension statistique de la structure linguistique et distinction phonétique/phonologie (communication faite à l'International Phonetic Sciences Congress, Miami), in: Hollen H. et P. (éds., 1979), Current Issues in the Phonetic Sciences, Amsterdam, John Benjamins.

ReY-Debove Josette, ReY Alain (éds., 2002), Le nouveau petit Robert, Paris, Dictionnaire Le Robert.

SCHOCH Marianne, DE SPENGLER Nina (1980), Rigueur et laxité en phonologie, La linguistique, vol. 16, no. 1 (Constance et variations), Paris, PUF, p. 105117.

\section{SUMMARY}

This study aims to characterize the concepts of centre and periphery with regard to different linguistic levels, phonemes, morphemes, "synthemes" and grammatical relations, from a double viewpoint, systemic relations/use, through various criteria, integration, distribution, information, etc. The distinction centre/periphery is briefly related to the dynamics of language development.

As a conclusion, the correlative concepts of centre and periphery cannot be defined as an absolute distinction, but as a variable and gradual one, which requires to be reconsidered in each situation where it is used. 